\title{
Eyelid eversion for visualisation of the upper eyelid lamellae: an anatomical cadaver study
}

\author{
Marcus M Marcet, ${ }^{1,2,3}$ Bradley N Lemke, ${ }^{4}$ Mark J Greenwald, ${ }^{2}$ Tamara R Fountain, ${ }^{5}$ \\ Steven Roth, ${ }^{6}$ Sander R Dubovy ${ }^{7}$
}

${ }^{1}$ Eye Institute, University of Hong Kong, Cyberport, Hong Kong

${ }^{2}$ Department of Surgery, University of Chicago, Chicago, Illinois, USA

${ }^{3}$ Department of Pathology, University of Chicago, Chicago, Illinois, USA

${ }^{4}$ Department of Ophthalmology, University of Wisconsin, Madison, Wisconsin, USA ${ }^{5}$ Department of Ophthalmology, Rush University Medical Center, Chicago, Illinois, USA ${ }^{6}$ Anesthesia and Critical Care, University of Chicago, Chicago, Illinois, USA

Bascom Palmer Eye Institute, University of Miami, Miami, Florida, USA

\section{Correspondence to}

Dr Marcus M Marcet, University of Hong Kong, Eye Institute, Rm 301, Blk B, Cyberport 4, 100 Cyberport Rd, Cyberport, Hong Kong; marcet@hku.hk

Presented at the Oculoplastics Paper Session, 26th Asia-Pacific Academy of Ophthalmology Congress in Sydney, Australia on 22 March 2011.

Accepted 15 June 2011

Published Online First

11 July 2011

\section{ABSTRACT}

Controversy persists in surgical eyelid anatomy despite the routine use of microanatomical examination in modern eyelid research. The aim of our study was to facilitate visualisation of upper eyelid anatomy by optimising the orientation of cadaveric specimens. We studied the anatomy of everted eyelids, providing an excellent histological view of the posterior approach to the eyelid commonly used in surgery. Non-traumatic separation of the eyelid lamellae provides a new view of the eyelid's lamellar nature. Further application of this model may enhance understanding of the multilayered aspect of the levator aponeurosis. The technique may improve intraoperative understanding of critical eyelid anatomy and promote safer and more effective eyelid surgery.

\section{INTRODUCTION}

The anatomical arrangement of the levator palpebrae superioris muscle (LPS), its anterior lamella (the levator aponeurosis (LA)) and its posterior lamella (the Müller muscle (MM)) have historically been incompletely understood. ${ }^{1}{ }^{2}$ Knowledge of LPS anatomy is essential for safe and effective eyelid surgery. The gold standard for examination of eyelid anatomy has been the cadaveric model, which is classically studied in the sagittal plane. However, limited visualisation due to dissection technique, ${ }^{3}$ distortion ${ }^{4}$ and the close proximity of eyelid structures ${ }^{5}$ has fuelled continued debate.

Given the limitations of using cadavers, some have tried newer imaging modalities, for example, surface coil $\mathrm{MRI}^{6}$ and ultrasound biomicroscopy, ${ }^{7}$ to further advance knowledge of surgical eyelid anatomy. Yet, in spite of its limitations, light microscopy study of cadaveric tissue generally provides superior resolution and remains one of the main approaches for study of the LPS and its lamellae. 55689

We report on how to optimise the classic use of cadaveric specimens by eversion of fresh upper eyelid tissue, secured by suture and formalin fixation, to non-invasively visualise the eyelid lamellae. We use the model to study the surgical microanatomy of the layers of the LA.

\section{METHODS}

Twenty-three orbits from 18 fresh-frozen cadaver heads were used. We everted the upper eyelids of 10 orbits from five heads (two male and three female; four Caucasian, one African-American; age range 54-82 years; mean age 69 years). To keep the eyelid everted during specimen handling and fixation, a 4-0 silk suture was placed through the grey line and secured above the eyebrow (figure 1). The eyelids of the remaining 13 orbits (four male and nine female; 13 Caucasian; age range 42-100 years; mean age 76 years) were left undisturbed as controls for anatomical comparison. The heads were fixed supine in $10 \%$ neutral buffered formalin for 3 weeks, exenterated and sutures removed. The orbits were sectioned parasagittally along the axis of the orbit and examined grossly. All specimens were cut in $6 \mu \mathrm{m}$ sections, stained with Masson trichrome and studied histologically. Elastic stain was used as a counterstain ${ }^{10}$ for the 10 everted and seven non-everted eyelids.

\section{RESULTS}

All eyelids maintained accurate positioning (figure 2A). The LA arose from the superior half of the LPS. The inferior half of the striated LPS fibres transitioned into the smooth-muscle fibres of the MM. A multilayered LA was well seen in all 10 everted eyelids (figure 2B,C) and five of 13 (38\%) noneverted eyelids. The remaining non-everted eyelids had an equivocal or poorly visible monolayer LA arrangement (eight of $13 ; 62 \%$ ). The lamellae in the everted eyelids were very clearly seen.

\section{DISCUSSION}

Our histological eyelid anatomy study is the first to be performed on the everted eyelid. Our approach is based on the eversion of fresh eyelid tissue, secured by suture and formalin fixation, with subsequent examination. The results revealed excellent exhibition of the lamellar characteristics of the eyelid anatomy. Eversion of the eyelid stretches the most posterior lamellae and relaxes the more anterior lamellae. It is the more anterior relaxed lamellae that are seen to separate from the taut posterior lamellae in our everted specimens.

Eyelid eversion is a commonly performed technique in clinical and surgical settings. Clinically, eversion is used for inspection of the palpebral conjunctiva, tarsus and fornix. Intraoperatively, the eyelid is everted for access to the posterior aspect of the eyelid. In some surgeries, such as the MMconjunctival resection ptosis procedure, even when a Desmarres retractor is used to maximally evert the eyelid, the retractor is released prior to closing of the clamp. ${ }^{11}$ Analysis of the everted eyelid sheds light on the orientation of the internal surgical anatomy and may lead to better understanding of common eyelid procedures. ${ }^{10}$

Cadaveric tissue is commonly used for the gross dissection and microscopic study of surgical eyelid 


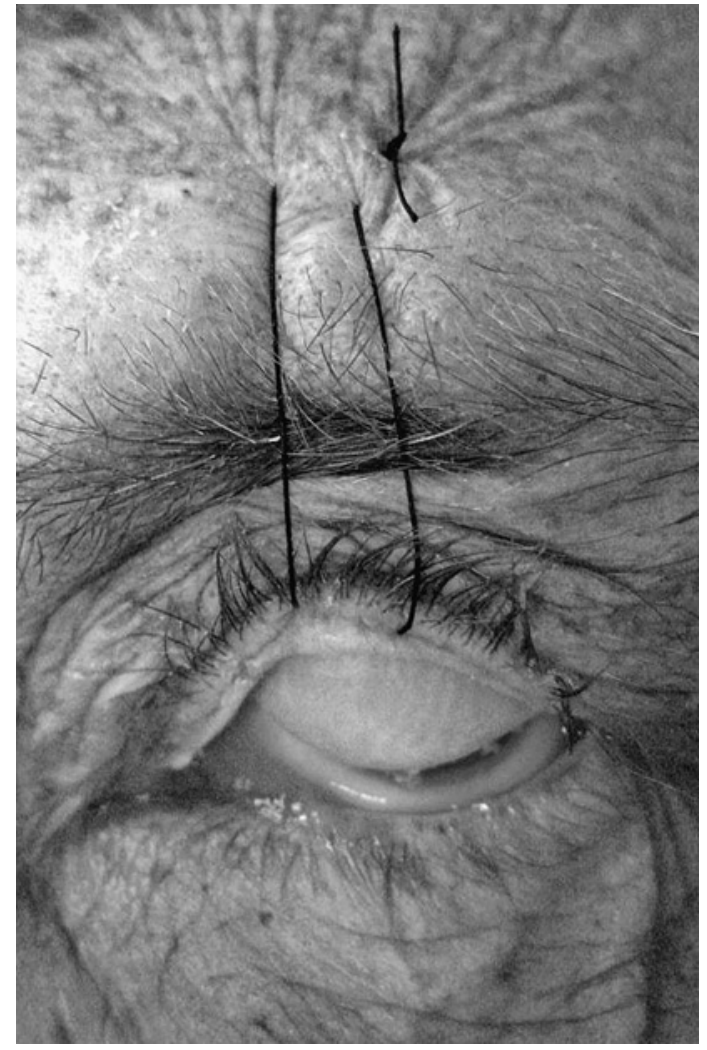

Figure 1 Anterior view of everted upper eyelid with retention suture.

anatomy, but dissection of specimens runs the risk of altering tissue planes. Furthermore, the close proximity and parallel course of the lamellae have caused difficulty in the study of eyelids in the classic sagittal orientation. ${ }^{5}$ Artefacts may occur with the positioning, fixation and sectioning of the delicate tissues. ${ }^{3} 4$ The limitations of the everted eyelid model potentially include the same artefacts of working with delicate tissues and thus the model is intended to broaden options rather than replace the classic approach.

In comparison with the classical approach, with eyelid eversion, the multiple layers of the LA separate. In the everted eyelid, the lamellae are more distinct because the superficial LA runs parallel with the tarsus, while the deep LA runs approximately perpendicular to the tarsus. The findings in our study are concordant with Anderson and Beard's 1977 description of a complex aponeurosis, with multiple layers having a 'fan of insertions' as shown in their grossly dissected eyelid specimens. ${ }^{8}$ The challenges of the classical orientation may have played a role in more recent histological descriptions of the layers of the LA, which include the presence of a double-layered LA in all Asian eyelids but only one of 11 Caucasian specimens. ${ }^{9}$ The everted eyelid model seems to more clearly demonstrate that the multilayered LA anatomy is shared not only by Asians, but also by African-Americans and Caucasians alike. However, an additional limitation of our study is that no Asian eyelids were examined and thus further studies using everted Asian eyelids would be necessary to exclude the possibility of anatomical variation. As common anatomy, it is interesting to consider that a multilayered LA may have a functional role in eyelid mechanics, rather than simply resulting in external phenotypic variation.

In summary, the everted eyelid model has several advantages over the classic sagittal orientation. First, the everted approach
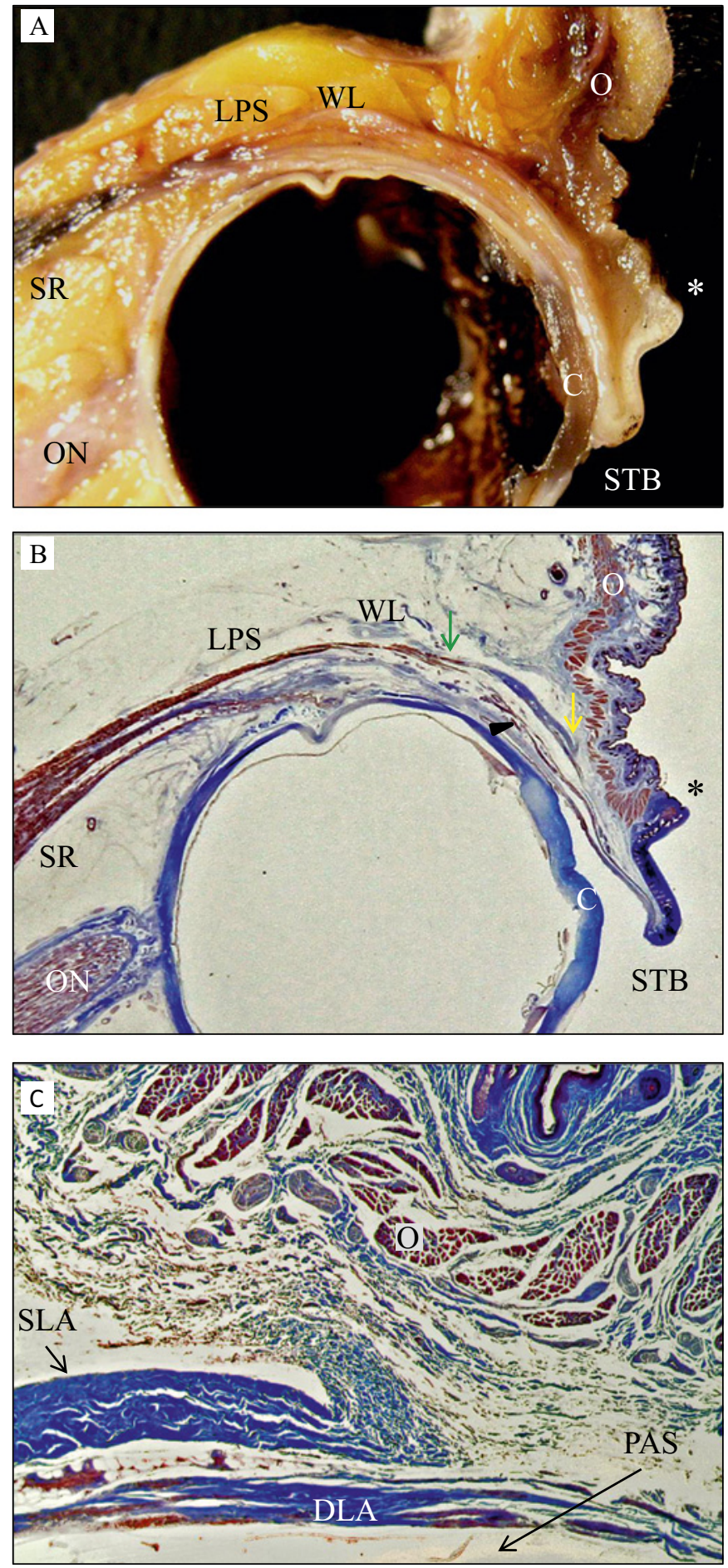

Figure 2 Parasagittal section $(A)$ and photomicrograph (Verhoeff-Masson trichrome, original magnification $\times 1$ ) (B) showing everted upper eyelid. Note that the superior tarsal border (STB) and eyelid margin (asterisk) are opposite to their normal anatomical positions. Vertical bifurcation of the striated muscle fibres of the levator palpebrae superioris (LPS, green arrow) into the multilayered levator aponeurosis (LA, yellow arrow) and Müller muscle (arrowhead) are demonstrated. Whitnall's ligament (WL), orbicularis oculi muscle (0), superior rectus muscle (SR), cornea (C) and optic nerve (ON) are also seen. The yellow arrow also designates the area shown in (C). (C) Photomicrograph (Verhoeff-Masson trichrome, original magnification $\times 40$ ) showing distinct superficial (SLA) and deep (DLA) aspects of the multilayered LA. The SLA is relatively relaxed and diverges approximately perpendicular to the DLA. The orbicularis oculi muscle $(0)$ and post-aponeurotic space (PAS) can also be seen. 
provides clinicoanatomical correlation with the frequent procedures that include eyelid eversion. The technique we describe is relevant to understanding the clinical and surgical anatomy of a commonly performed manoeuvre. Second, the technique allows non-invasive separation of the eyelid lamellae, which permits improved visualisation of the lamellar aspect of eyelid retractor anatomy; an example is that it allowed us to establish the consistency of multilayered LA anatomy. Third, the eyelid eversion model provides a fresh perspective on anatomical relationships that have long been debated by surgeons. While the everted model is intended as an alternative rather than a replacement for the classic orientation, the broader benefits of facilitating further studies of surgical eyelid anatomy make the model particularly significant.

Acknowledgements We thank Robin Bozarth and the staff of the Medical Education and Research Institute, Memphis, Tennessee, and Fran Lietz and the staff of the Departments of Anatomy, Surgery and Pathology, University of Chicago, for their assistance.

Funding Section of Ophthalmology, Department of Surgery, University of Chicago, Chicago, IL, USA.

\section{Competing interests None.}

Ethics approval The study was performed in compliance with the University of Chicago Institutional Review Board's policy on research on decedents. In addition, the study conformed to the principles of the Declaration of Helsinki.
Contributors As guarantor of the paper, MMM, accepts full responsibility for the conduct of the study, had access to the data and controlled the decision to publish

Provenance and peer review Not commissioned; externally peer reviewed.

\section{REFERENCES}

1. Whitnall SE. Anatomy of the Human Orbit and Accessory Organs of Vision. Reprint of 1932 edition. Huntington, NY: Robert E. Krieger Publishing, 1979:115-64.

2. Berke RN, Wadsworth JA. Histology of levator muscle in congenital and acquired ptosis. AMA Arch Ophthalmol 1955;53:413-28.

3. Dortzbach RK, Sutula FC. Involutional blepharoptosis. A histopathological study. Arch Ophthalmol 1980;98:2045-9.

4. Siegel R. Surgical anatomy of the upper eyelid fascia. Ann Plast Surg 1984;13:263-73.

5. Haramoto U, Kubo T, Tamatani $\mathrm{M}$, et al. Anatomic study of the insertions of the levator aponeurosis and Müller's muscle in oriental eyelids. Ann Plast Surg 2001;47:528-33.

6. Meyer DR, Linberg JV, Wobig JL, et al. Anatomy of the orbital septum and associated eyelid connective tissues. Implications for ptosis surgery. Ophthal Plast Reconstr Surg 1991;7:104-13.

7. Demirci H, Nelson CC. Ultrasound biomicroscopy of the upper eyelid structures in normal eyelids. Ophthal Plast Reconstr Surg 2007;23:122-5.

8. Anderson RL, Beard C. The levator aponeurosis. Arch Ophthalmol 1977;95:1437-41.

9. Kakizaki H, Madge SN, Malhotra R, et al. The levator aponeurosis contains smooth muscle fibers: new findings in Caucasians. Ophthal Plast Reconstr Surg 2009;25:267-9.

10. Marcet MM, Setabutr P, Lemke BN, et al. Surgical microanatomy of the Müller muscle-conjunctival resection ptosis procedure. Ophthal Plast Reconstr Surg 2010;26:360-4.

11. Putterman AM, Urist MJ. Müller muscle-conjunctival resection. Technique for treatment of blepharoptosis. Arch Ophthalmol 1975:7:263-6.
Advancing Postgraduates. Enhancing Healthcare.

The Postgraduate Medical Journal is dedicated to advancing the understanding of postgraduate medical education and training.

- Acquire the necessary skills to deliver the highest possible standards of patient care

- Develop suitable training programmes for your trainees

- Maintain high standards after training ends

Published on behalf of the fellowship for Postgraduate Medicine

FOR MORE DETAILS OR TO SUBSCRIBE, VISIT THE WEBSITE TODAY

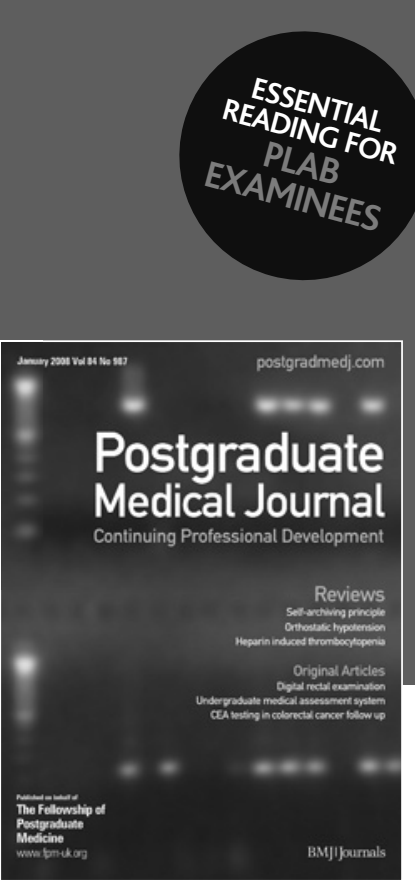

BMJIJournals 